\title{
DISTRIBUIÇÃO ESPACIAL DA CTC E DA RELAÇÃO DOS MACRONUTRIENTES EM UM LATOSSOLO VERMELHO-AMARELO CULTIVADO COM CAFEEIRO
}

\author{
Leandro da Silva Almeida ${ }^{1}$,Ednaldo Carvalho Guimarães ${ }^{2}$ \\ ${ }^{1}$ Doutorando em Agronomia, Instituto de Ciências Agrarias, Universidade Federal de Uberlândia (UFU), \\ Uberlândia, Minas Gerais. E-mail: almeidalean26@gmail.com \\ ${ }^{2}$ Professor Titular, Faculdade de Matemática, Universidade Federal de Uberlândia (UFU), Uberlândia, Minas \\ Gerais.
}

RESUMO: O cafeicultor sempre busca maximizar a sua produtividade, isto requer a compreensão dos fatores que a influência, dentre os vários fatores que influenciam o rendimento do cafeeiro, a nutrição, com certeza é de suma importância, e está, é afetada pelo balanço dos nutrientes no solo. Assim, o objetivo deste estudo foi analisar e descrever o comportamento espacial da capacidade de troca de cátions em pH 7 (CTC) e das relações $\mathrm{Ca} / \mathrm{T} ; \mathrm{Mg} / \mathrm{T} ; \mathrm{K} / \mathrm{T}$ e da matéria orgânica (M.O) em um Latossolo Vermelho-Amarelo cultivado com cafeeiro. $\mathrm{O}$ experimento foi realizado em uma área de 65 ha cultivada com cafeeiro arábica (Coffea arabica L.), localizada no município de Araguari-MG. Foi utilizada uma malha não sistemática de um ponto por hectare, totalizando 65 pontos georeferenciados. As amostras de solo foram retiradas na projeção da copa do cafeeiro na profundidade de 0-0,2 $\mathrm{m}$. Foram ajustados os semivariogramas para a CTC e as relações $\mathrm{Ca} / \mathrm{T} ; \mathrm{Mg} / \mathrm{T} ; \mathrm{K} / \mathrm{T}$ e $\mathrm{Ca} / \mathrm{Mg}$, sendo que as relações $\mathrm{Mg} / \mathrm{T}, \mathrm{K} / \mathrm{T}$ e $\mathrm{Ca} / \mathrm{Mg}$ apresentaram moderada dependência espacial e a CTC e a relação $\mathrm{Ca} / \mathrm{T}$ uma forte dependência, já a M.O apresentou efeito pepita puro. Os modelos de semivariogramas ajustados pela semivariância dos dados foram: o exponencial para a CTC, o esférico para a relação $\mathrm{Ca} / \mathrm{T}$ e o gaussiano para as demais relações. A interpolação dos dados foi realizada por krigagem ordinária, a exceção da M.O. que foi através do inverso do quadrado da distância. O maior alcance prático foi da CTC do solo $600 \mathrm{~m}$, o menor da relação $\mathrm{Ca} / \mathrm{Mg}$ de $287,80 \mathrm{~m}$, já as relações $\mathrm{Ca} / \mathrm{T}, \mathrm{Mg} / \mathrm{T}$ e $\mathrm{K} / \mathrm{T}$ apresentaram alcances de 581,86, 575,60 e 467,70 m respectivamente.

Palavras-chave: Krigagem ordinária. Planejamento de adubação. Equilíbrio entre nutrientes. Variabilidade espacial.

\section{SPATIAL DISTRIBUTION OF THE CTC AND THE RELATIONSHIP OF MACRONUTRIENTS IN A RED-YELLOW LATOSOL CULTIVATED WITH COFFEE CROP}

\begin{abstract}
The coffee grower always seeks to maximize his productivity, this requires understanding the factors that influence, among the several factors that influence coffee yield, nutrition, it sure is a very important and is, is affected by the balance of nutrients in the soil. Thus, the objective of this study was to analyze and describe the spatial behavior of
\end{abstract}

Cultura Agronômica, Ilha Solteira, v.26, n.4, p.625-639, 2017 
the cation exchange capacity at $\mathrm{pH} 7$ (CTC) and $\mathrm{Ca} / \mathrm{T}$ ratios; $\mathrm{Mg} / \mathrm{T} ; \mathrm{K} / \mathrm{T}$ and organic matter (O.M.) in a Red-Yellow Latosol cultivated with coffee. The experiment was carried out in an area of 65 ha cultivated with coffee arabica (Coffea arabica L.), located in the municipality of Araguari-MG. A non-systematic mesh of one point per hectare was used, totaling 65 georeferenced points. Soil samples were removed in the projection of the coffee cup in the 0-0.2 m depth. Semivariograms were adjusted for CTC and for rations $\mathrm{Ca} / \mathrm{T}$; $\mathrm{Mg} / \mathrm{T} ; \mathrm{K} / \mathrm{T}$ and $\mathrm{Ca} / \mathrm{Mg}$, and the $\mathrm{Mg} / \mathrm{T}, \mathrm{K} / \mathrm{T}$ and $\mathrm{Ca} / \mathrm{Mg}$ ratios showed moderate spatial dependence and the CTC and the Ca / T ratio a strong dependence, whereas the O.M. had pure nugget effect. The semivariograms models adjusted by the data semivariance were: the exponential for the CTC, the spherical for the $\mathrm{Ca} / \mathrm{T}$ ratio and the Gaussian for the other relations. The interpolation of the data was performed by ordinary kriging, with the exception of O.M. which was through the inverse of the square of the distance. The greatest practical range was the CTC of the $600 \mathrm{~m}$ soil, the lowest of the $\mathrm{Ca} / \mathrm{Mg}$ ratio of $287.80 \mathrm{~m}$, while the $\mathrm{Ca} / \mathrm{T}, \mathrm{Mg} / \mathrm{T}$ and $\mathrm{K} / \mathrm{T}$ ratios had a range of $581.86,575.60$ and $467.70 \mathrm{~m}$ respectively.

Key words: Ordinary Kriging. Fertilization Planning. Nutrient Balance. Spatial Variability.

\section{INTRODUÇÃO}

O destaque da cafeicultura no contexto socioeconômico brasileiro está na sua amplitude e na riqueza gerada, pois é cultivado em mais de 2,2 milhões de hectares no país, com produção estimada de 45,5 milhões de sacas para a safra 2017, sendo que Minas Gerais deve produzir aproximadamente $60 \%$ deste total, ou seja, uma produção estimada de mais de 26 milhões de sacas (CONAB, 2017).

Em 2016 as exportações de café arábica cru representaram 2,61\% do total das receitas obtidas com as exportações brasileiras, foram exportadas mais de 29,7 milhões de sacas, que geraram divisas no valor de U\$ 4,8 bilhões, acrescentando também as exportações de café conilon, e dos cafés beneficiados (torrado e solúvel) o valor com as exportações do agronegócio café representaram mais de U\$ 5,4 bilhões, num total de 34,2 milhões de sacas (CECAFE, 2017).

O cafeicultor atual está em busca de maximizar a produção, para isso é preciso compreender os fatores que a influenciam, como os caminhos para aumentar a produtividade de forma sustentável. Dentre os vários fatores que influenciam o rendimento, a nutrição de plantas assume grande importância para a maioria dos solos brasileiros, devido a baixa fertilidade natural (FONTES; PEREIRA, 2003).

Por este motivo, manejar a fertilidade do solo através do emprego eficiente de adubos e corretivos, independentemente da origem (orgânico, mineral, organomineral) é o principal fator para o incremento das produtividades nas culturas (LOPES; GUILHERME, 2007). Sendo que os atributos químicos do solo possuem uma distribuição bastante complexa, 
decorrentes do material de origem e das alterações geradas pelos sistemas e de manejo adotados (SOUZA et al., 2008).

Meurer et al. (2006), estudando os fenômenos de sorção em solos, relatam que os Latossolos, são intemperizados, com caráter ácido, de baixa capacidade de troca de cátions (CTC). Sendo que nestes solos, segundo Rosolen et al. (2012), cerca de 50\% ou mais da capacidade de trocas de cátions é proveniente da M.O. do solo.

Para qualidade da nutrição do cafeeiro, faz-se necessário ressaltar a relação entre os nutrientes, pois a demasia de um nutriente pode inibir a absorção do outro, por antagonismo ou pela competição dos sítios de absorção (NOVAIS et al., 2007), e ocasionando deficiências e consequentemente perdas nas produtividades causado pelo desiquilíbrio nutricional ocorrido, além disso a planta ainda pode ficar mais suscetível ao ataque de insetos e fungos (CARVALHO et al., 2013).

A agricultura de precisão tem dentre os seus objetivos, entender a dinâmica do comportamento dos atributos químicos do solo, no espaço, por meio da quantificação da variabilidade espacial, buscando assim, uma melhor eficiência da aplicação de fertilizantes e corretivos na agricultura (MOLIN; CASTRO, 2008), além de trazer possíveis benefícios ambientais, pois permite o uso racional dos recursos naturais e evita aplicações de produtos em excesso o que poderia levar à contaminação do solo e de recursos hídricos pelos processos de lixiviação e também escorrimento superficial (ALMEIDA; GUIMARÃES, 2016).

O comportamento da variabilidade espacial dos atributos do solo pode ser determinado tanto por estatística clássica, quanto por geoestatística, no entanto, se os dados não revelam uma variação completamente aleatória, a geoestatística torna se uma ferramenta imprescindível, para poder assim descrever o comportamento destes atributos na área (ALMEIDA; GUIMARÃES, 2016).

O objetivo deste trabalho foi analisar e descrever o comportamento espacial da capacidade de troca de cátions em pH 7,0 (CTC) e das relações $\mathrm{Ca} / \mathrm{T} ; \mathrm{Mg} / \mathrm{T} ; \mathrm{K} / \mathrm{T}$ e $\mathrm{Ca} / \mathrm{Mg}$, em um Latossolo Vermelho-Amarelo cultivado com cafeeiro no município de AraguariMG.

\section{MATERIAL E MÉTODOS}

O trabalho foi desenvolvido na Fazenda Brasil, município de Araguari-MG, situadas nas coordenadas geográficas: $-18^{\circ} 32 ' 55.99 "$ ao sul da linha do Equador, $-48^{\circ} 24^{\prime} 10.46^{\prime \prime}$ a oeste do meridiano de Greenwich. Na área de estudo são cultivados 65 hectares de café (Coffea arabica L.), variedade Mundo Novo, plantados em 1989. A altitude é de 940 metros e o solo classificado como Latossolo Vermelho-Amarelo com $38 \%$ de argila e $21 \%$ de silte. Além disso, a área cultivada é irrigada por gotejamento com uso de fertirrigação. $\mathrm{O}$ clima predominante da região é do tipo Cwa, ou seja, clima subtropical com o inverno seco e o verão quente segundo a classificação climática de Köppen (1948).

Cultura Agronômica, Ilha Solteira, v.26, n.4, p.625-639, 2017 
Nessas áreas, foram estabelecidas malhas de um ponto por hectare, totalizando 65 pontos georeferenciados. As amostras de solo, foram coletadas no mês de setembro de 2014, foram retiradas na profundidade de $0,00-0,20 \mathrm{~m}$, respeitando a projeção do dossel do cafeeiro para CTC, da relação $\mathrm{Ca} / \mathrm{Mg}, \mathrm{Ca} / \mathrm{T}, \mathrm{Mg} / \mathrm{T}$ e K/T . Também foi analisada a M.O. do solo pela sua íntima relação com a CTC. Os resultados das análises foram obtidos de acordo com a Embrapa (2011). As análises foram realizadas no Laboratório de Análise de Solo (LABAS) da Universidade Federal de Uberlândia.

Foram calculadas pela análise exploratória dos dados através das médias, medianas, variâncias, máximos e mínimos, desvios padrões dos atributos do solo analisados, a fim de conhecer o comportamento geral dos dados e verificar possíveis valores atípicos.

Foi realizada também a analise descritiva espacial, verificando tendência dos dados, por meio de gráficos de dispersão para as direções leste $(\mathrm{x})$ e norte (y) de cada atributo do solo, também foi gerado os mapas de localização dos pontos amostrais na área, e por fim, os histogramas dos atributos, a fim de, verificar o comportamento de cada variável na área e a assimetria ou simetria dos dados respectivamente, que pode interferir na modelagem espacial e no processo de interpolação pela krigagem (YAMAMOTO; LANDIM, 2013).

Para os atributos que apresentaram tendência em pelo menos em uma das direções, as análises seguintes foram realizadas com os resíduos dos dados obtidos por meio da regressão linear, sendo que nestes casos específicos, ao final do processo, ou seja, depois de realizar a krigagem dos resíduos, os dados originais foram retomados através da equação da regressão a fim de gerar o mapa temático do atributo para a área.

A modelagem da dependência espacial foi realizada através da semivariância dos dados, que é uma medida de dissimilaridade dos dados. O cálculo foi realizado pela formula indicada por Matheron (1963) conforme apresentado na Equação 1.

$$
\bar{Y}(h)=\frac{1}{2 N(h)} * \sum^{N(h)}[Z(s)-Z(s+h)]^{2}
$$

Onde: $\bar{\Upsilon}(h)$ é a semivariância estimada para a distância $h, N(h)$ é o número de pares da diferença da variável $\mathrm{Z}$ medida na posição s e s+h (posição + distância).

Os parâmetros do semivariograma foram estimados a sentimento, conforme proposto por Vieira et al. (1983), associados a validação cruzada, ou seja, foram ajustados vários modelos e verificada a qualidade dos ajustes por meio da validação cruzada, sendo selecionados aqueles que melhor representavam os atributos em campo. O processo de validação foi realizado conforme descrito em Soares (2006).

Após o ajuste do modelo teórico do semivariograma, avaliou-se o grau de dependência espacial (Equação 2), conforme proposto por Cambardella et al. (1994).

$$
\mathrm{GD}=\frac{\mathrm{Co}}{\mathrm{Co}+\mathrm{C}}
$$

Onde: $\mathrm{GD}=$ grau de dependência espacial; $\mathrm{Co}=$ Efeito Pepita; $\mathrm{C}=$ contribuição $(\mathrm{Co}+\mathrm{C}=$ patamar $)$. 
Sendo: GD $<0,25$ Forte; $\geq 0,25$ GD $\leq 0,75$ Moderada; e, GD > 0,75 Fraca

Após estas verificações para os micronutrientes que apresentaram dependência espacial aplicou-se a krigagem ordinária para a interpolação (YAMAMOTO; LANDIM, 2013). Quando os atributos não apresentaram dependência espacial, ou seja, apresentaram efeito pepita puro, estes foram interpolados pelo método do inverso do quadrado das distâncias, que, conforme Krajewski; Gibbs (2001), é o método de interpolação por estatística clássica mais assertivo.

Todas as análises geoestatística foram realizadas com o auxílio do programa $\mathrm{R}$ versão 3.2.1 (R Development Core Team, 2015), utilizando o pacote de dados geoR (RIBEIRO JÚNIOR; DIGGLE, 2001).

Para a classificação das faixas obtidas nos mapas de padrões espaciais, foram utilizados os critérios relativos a cada atributo do solo conforme proposto Alvarez V. et al. (1999), no Manual de Recomendações do Uso de Fertilizantes e Corretivos em Minas Gerais ( $5^{\mathrm{a}}$ Aproximação).

\section{RESULTADOS E DISCUSSÃO}

As estatísticas descritivas dos atributos químicos do Latassolo Vermelho-Amarelo cultivado com cafeeiro são apresentadas na Tabela 1. Observe que a CTC do solo está dentro do limite considerado mediano para os solos no estado de Minas Gerais que é de 4,31 a 8,60 $\mathrm{cmol}_{\mathrm{c}} \mathrm{dm}^{-3}$ (ALVARES V. et al., 1999), e que a sua amplitude na área é baixa, apresentado assim uma pequena variância. Rosolen et al. (2012), evidenciaram que cerca de $50 \%$ da CTC do solo na região de cerrado é proveniente da M.O., e neste mesmo sentido, Novais et al. (2007), afirmam que nos solos tropicais intemperizados até $80 \%$ da CTC é oriunda da M.O. que neste solo oscila de 1,08 a 3,31\% (Tabela 1).

Tabela 1. Estatística descritiva dos atributos do solo com cultura do café da Fazenda, município de Araguari-MG, no ano de 2014.

\begin{tabular}{llllllll}
\hline Atributos & Méd. & Mediana & DP & Var $^{2}$ & Máx. $^{3}$ & Min. $^{4}$ & CV $^{5}$ \\
\hline C.T. de Cations(T) $\left(\mathrm{cmol}_{\mathrm{c}} \mathrm{dm}^{-3}\right)$ & 6,22 & 6,21 & 0,43 & 0,19 & 7,10 & 5,41 & 7,00 \\
Relação Ca/Mg (\%) & 3,37 & 3,33 & 0,38 & 0,14 & 4,50 & 2,67 & 11,2 \\
Relação Ca/T (\%) & 34,41 & 35,35 & 6,80 & 46,25 & 47,48 & 15,61 & 19,2 \\
Relação Mg/T (\%) & 10,32 & 10,37 & 2,19 & 4,80 & 14,47 & 4,26 & 21,2 \\
Relação K/T (\%) & 8,34 & 7,09 & 3,06 & 9,35 & 15,51 & 3,68 & 36,7 \\
Matéria Orgânica (\%) & 2,14 & 2,17 & 0,59 & 0,35 & 3,31 & 1,08 & 27,5 \\
\hline
\end{tabular}

${ }^{1}$ D.P=desvio padrão; ${ }^{2}$ Var.= Variância ${ }^{3} \mathrm{Max}=$ Máximo; ${ }^{4} \mathrm{Min}=$ mínimo; ${ }^{5} \mathrm{CV}=$ coeficiente de variação.

A relação considerada ideal para $\mathrm{Ca} / \mathrm{Mg}$ no cafeeiro é de 3 a 5:1 (ALVAREZ V. et al., 1999). $\mathrm{Na}$ área do estudo, foi diagnosticada a relação $\mathrm{Ca} / \mathrm{Mg}$ média de 3,3:1, estando assim dentro da faixa ideal.

Cultura Agronômica, Ilha Solteira, v.26, n.4, p.625-639, 2017 
Segundo Medeiros et al. (2008) e Matiello et al. (2006), os valores da saturação de $\mathrm{Ca}^{+2}$ na CTC do solo para uma boa nutrição das plantas, variam de 40 a $50 \%, \mathrm{Mg}^{+2}$ entre 15 a $20 \%$ e os referentes a saturação de $\mathrm{K}^{+}$variam entre 3 a $5 \%$. Verificou-se que, na área em estudo (Tabela 1), as relações dos nutrientes na CTC não estão condizentes com estes intervalos de equilíbrios, sendo respectivamente em média de $34,41 \% ; 10,32 \%$ e $8,34 \%$, para $\mathrm{Ca}^{+2}, \mathrm{Mg}^{+2}$ e K , o que compromete a absorção de nutrientes pelo cafeeiro, neste caso principalmente o Mg (NOVAIS et al., 2007).

O coeficiente de variação oscilou entre $7 \%$ e $36,7 \%$, sendo estes valores extremos observados para CTC e a relação entre K/T respectivamente (Tabela 1). Adotando o critério de classificação proposto por Gomes (1984) para esta medida estatística de dispersão, a CTC teve baixa variação (7\%), as relações $\mathrm{Ca} / \mathrm{Mg}$ e Ca/T tiveram média variação $(11,2$ e $19,2 \%$ respectivamente), a matéria orgânica (MO) e a relação de $\mathrm{Mg} / \mathrm{T}$ tiveram alta variação (27,5 e 21,2\% respetivamente) e por fim a relação do $\mathrm{K} / \mathrm{T}$ teve uma variação considerada muito alta. Um alto coeficiente de variação segundo Carvalho et al. (2003) é esperado quando os dados apresentam grande amplitude nos valores conforme também se revela os valores de mínimo e máximo (Tabela 1). Destaca-se ainda que apesar de variabilidade relativamente alta para a relação $\mathrm{K} / \mathrm{T}$, as estatísticas média e mediana possuem valores próximos entre si, portanto, ocorre uma tendência simétrica na distribuição dos dados e, de acordo com Yamamoto e Landim (2013), para aplicação do estimador de Matheron (1963) é recomendável que a variável não apresente assimetria acentuada.

$\mathrm{Na}$ análise descritiva espacial foram elaborados os mapas de distribuição dos elementos na área do estudo e também realizada a verificação de tendência dos dados nas direções leste (x) e norte (y) e os histogramas dos conjuntos de dados (Figura 1).

Por meio da verificação dos quartis (Q1, Q2 e Q3) e dos gráficos de dispersão para os sentidos $\mathrm{x}$ e y verifica-se, que os atributos do solo, a exceção da relação $\mathrm{K} / \mathrm{T}$, não apresentaram comportamentos semelhantes em alguma posição na área, ou seja, verificou a ausência de tendência para os atributos: $\mathrm{CTC} ; \mathrm{Ca} / \mathrm{Mg} ; \mathrm{Ca} / \mathrm{T} ; \mathrm{Mg} / \mathrm{T}$ e M.O. A tendência não é fator limitante na análise dos semivariogramas, entretanto, quando está presente faz se necessária à transformação dos dados para executar a análise (SOARES, 2006).

Os histogramas (Figura 1) mostram, que não ocorrem assimetria acentuada para essas variáveis. Esses fatos sugerem a não necessidade de transformação de dados para a aplicação da metodologia de análise geoestatística usando o estimador de Matheron (VIEIRA et al., 1983). Mais importante que a normalidade dos dados é a ocorrência do efeito proporcional em que a média e a variância dos dados não sejam constantes na área de estudo, tendo em vista que os semivariogramas apresentam patamares bem definidos (CAVALCANTE et al., 2007). 

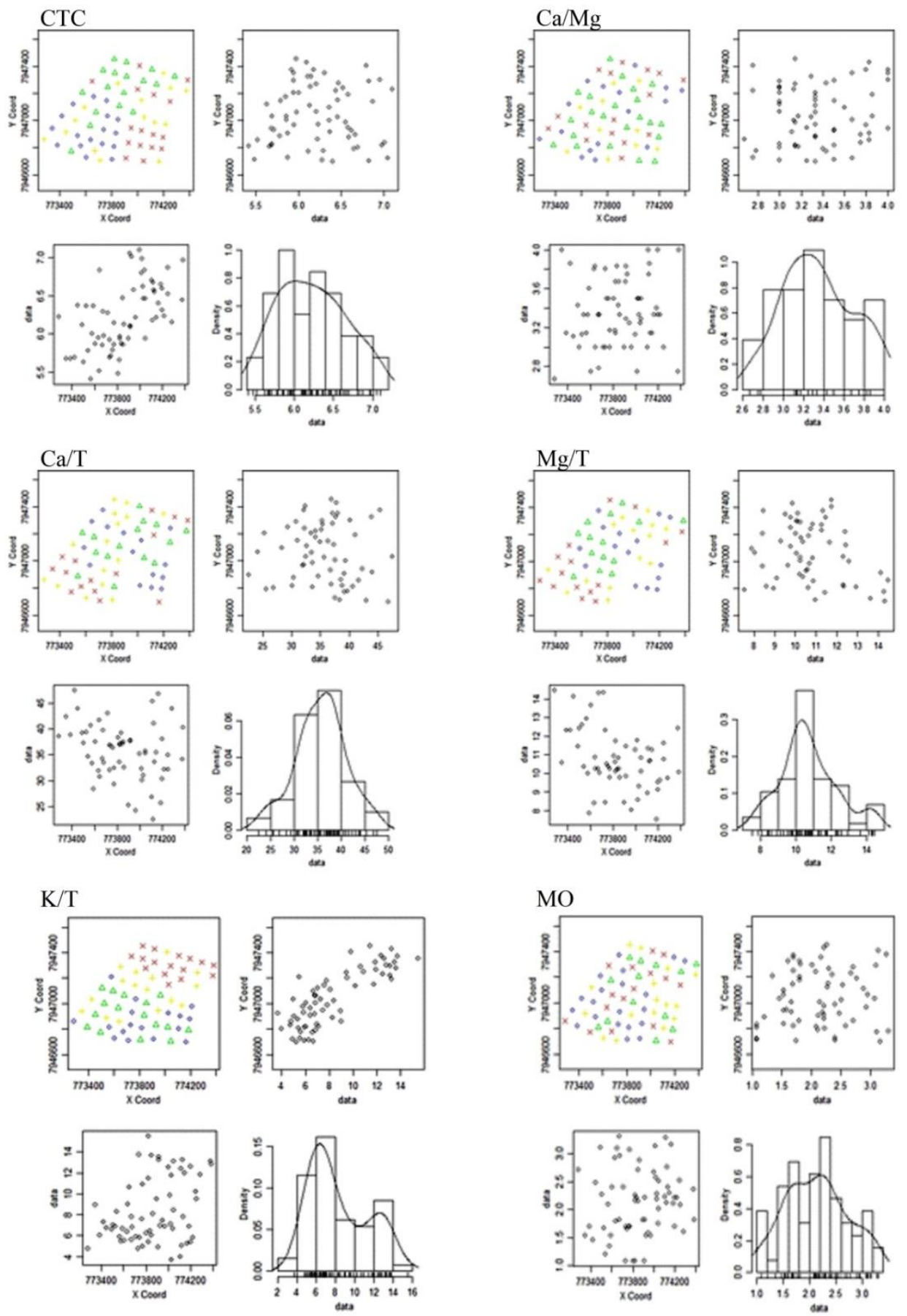

Figura 1. Mapas de posição dos pontos amostrados de acordo com os quartis (classes definidas na sequência de tamanhos das representações: Min |-- Q1; Q1 |-- Q2; Q2 |-- Q3; Q3 |-- Max). Dispersão sentido x e y e histograma dos atributos químicos do solo nos quais é possível observar a curtose e assimetria dos dados.

Para a relação K/T que apresentou tendência na direção norte (y), utilizou os resíduos dos dados obtidos por meio de regressão linear, para modelar a dependência espacial. O 
modelo de regressão linear que descreve a tendência para a relação $\mathrm{K} / \mathrm{T}$ é apresentado na equação 3.

$$
y=0,0028 x-2.188,73\left(\mathrm{R}^{2}=82 \%(0,82)\right)
$$

Sendo assim foram usados os resíduos da relação K/T em todas as fases dos cálculos, só retomando aos valores originais, ou seja, ao valor real do elemento, para a geração do mapa temático destes elementos na área.

CTC

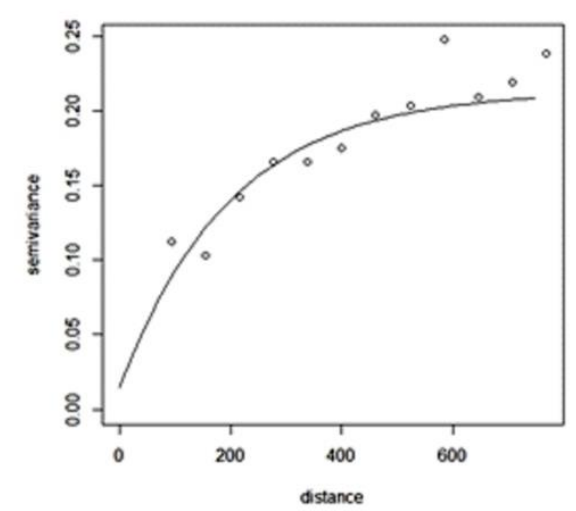

$\mathrm{Ca} / \mathrm{T}$

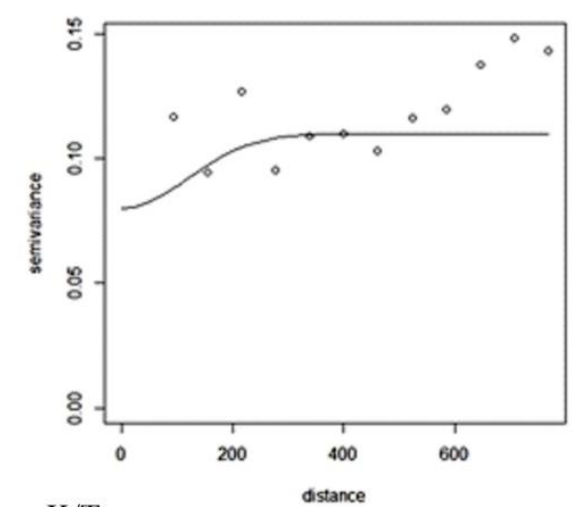

K/T

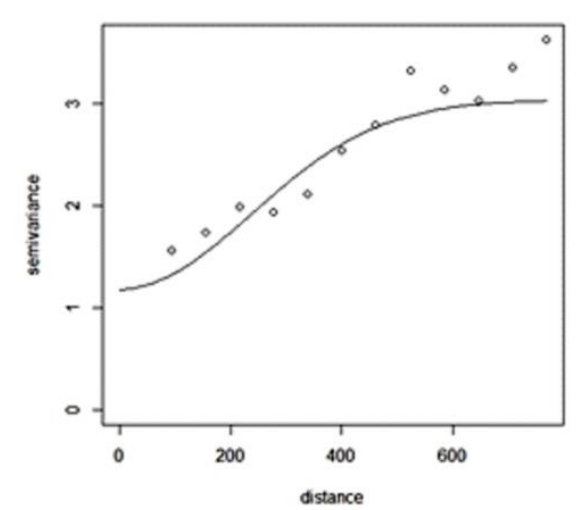

$\mathrm{Ca} / \mathrm{Mg}$

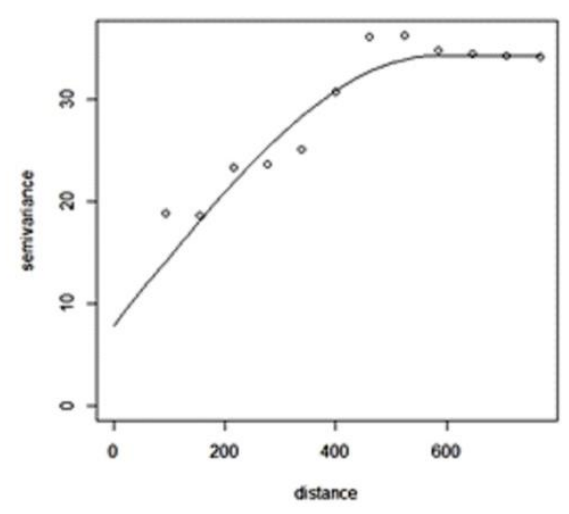

$\mathrm{Mg} / \mathrm{T}$

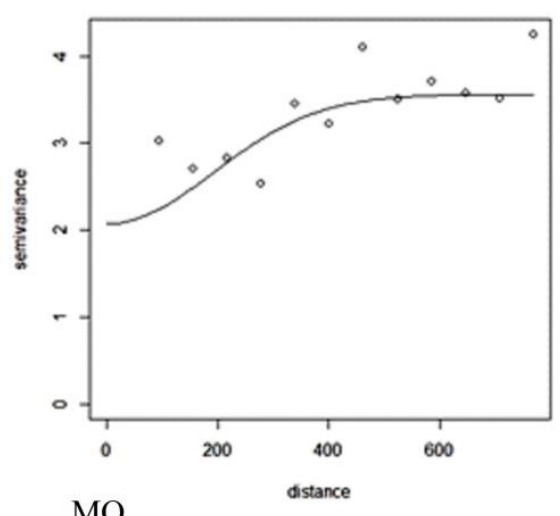

MO

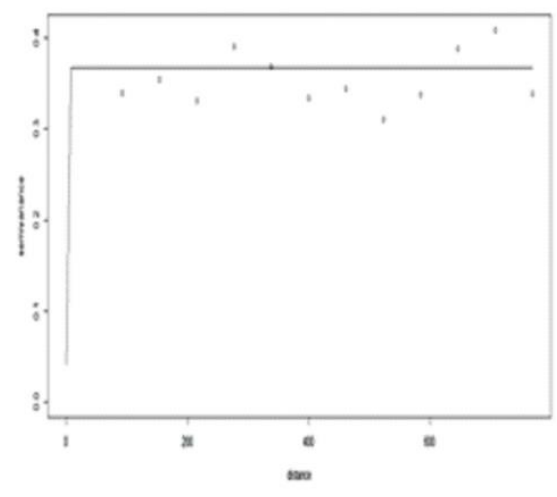

Figura 2. Semivariogramas ajustados para a CTC do solo a pH 7,0 (CTC), a relação entre os macronutrientes $(\mathrm{Ca} / \mathrm{Mg}$; $\mathrm{Ca} / \mathrm{T} ; \mathrm{Mg} / \mathrm{T}$ e $\mathrm{K} / \mathrm{T})$ e para a matéria orgânica do solo (MO). 
Para os atributos do solo estudado, foram ajustados os modelos de semivariogramas (Figura 2) sendo que todos os modelos foram ajustados a sentimento e selecionados aqueles que apresentaram melhores parâmetros baseados na validação cruzada (Tabela 2) (VIEIRA et al., 1983; YAMAMOTO; LAMDIM, 2013).

Tabela 2. Modelos, parâmetros, relação $\mathrm{Co} / \mathrm{Co}+\mathrm{C}$ e grau da dependência espacial dos semivariogramas ajustados para a CTC do solo e a relação entre os macronutrientes.

\begin{tabular}{lllllll}
\hline \multicolumn{1}{c}{ Atributo } & \multicolumn{1}{c}{ Modelo } & $\mathrm{C}$ & $\mathrm{C} 0$ & Alcance Prático & $\mathrm{Co} / \mathrm{Co}+\mathrm{C}^{\mathbf{1}}$ & \multicolumn{1}{c}{$\mathrm{GD}^{\mathbf{3}}$} \\
\hline $\mathrm{CTC}$ & Exponencial & 0,20 & 0,02 & 600,00 & 0,07 & Forte \\
$\mathrm{Ca} / \mathrm{Mg}$ & Gaussiano & 0,03 & 0,08 & 287,80 & 0,73 & Moderado \\
$\mathrm{Ca} / \mathrm{T}$ & Esférico & 26,49 & 7,85 & 581,86 & 0,23 & Forte \\
$\mathrm{Mg} / \mathrm{T}$ & Gaussiano & 1,86 & 1,18 & 575,60 & 0,39 & Moderado \\
$\mathrm{K} / \mathrm{T}^{2}$ & Gaussiano & 1,49 & 2,07 & 467,70 & 0,58 & Moderado \\
$\mathrm{MO}$ & $\mathrm{EPP}^{4}$ & ---- & ---- & ---- & ---- & Ausente \\
\hline${ }^{1} \mathrm{Co}=$ efeito pepita; $\mathrm{C}=$ contribuição; Co $+\mathrm{C}=$ patamar; ${ }^{1}$ método de Cambardella et al. (1994) ${ }^{2}$ modelo \\
ajustado com resíduos; ${ }^{3} \mathrm{GD}=$ Grau de dependência; ${ }^{4} \mathrm{EPP}=$ efeito pepita puro.
\end{tabular}

A M.O. não apresentou dependência espacial para as distâncias amostradas, apresentado assim um comportamento aleatório na área em estudo (efeito pepita puro), fato que segundo Lima et al. (2010) é pode ser devido a menor distância malha (77 m) não ter sido suficiente para determinar a dependência espacial. Para este atributo modelagem do padrão de distribuição espacial dos dados foi realizada pelo método do inverso do quadrado das distancias (IQD).

Verifica-se que os atributos do solo CTC e a relação Ca/T (Tabela 2) apresentaram forte grau de dependência espacial (GD <0,25). Oliveira et al. (2015), também encontraram forte dependência espacial para a CTC do solo cultivado com forrageira, entretanto do modelo de semivariograma ajustado foi o gaussiano. As relações $\mathrm{Ca} / \mathrm{Mg}, \mathrm{Mg} / \mathrm{T}$ e $\mathrm{K} / \mathrm{T}$ apresentaram moderada dependência espacial.

Verifica-se também que o alcance prático máximo foi a CTC $(600 \mathrm{~m})$, o mínimo foi da relação $\mathrm{Ca} / \mathrm{Mg}$ que foi de 287,80, apresentado também um grau de dependência menor, (GD=0,73), mesmo assim com um alcance expressivo, já que a distância mínima entre os pontos na área foi de $77 \mathrm{~m}$, isto é importante, pois baixos valores de alcance podem influir negativamente na qualidade das estimativas, uma vez que poucos pontos são usados para realização da interpolação na estimativa de valores em locais não medidos (LIMA et al. 2010).

No mapa de padrão espacial da CTC (Figura 3) está demonstrado sua distribuição em quatro classes na área, oscilando entre $5,5 \mathrm{a}^{7,0} \mathrm{cmol}_{\mathrm{c}} \mathrm{dm}^{-3}$ assim é possível identificar as faixas de manejo diferentes. Essas faixas são importantes principalmente para o planejamento do parcelamento dos fertilizantes, e na indicação de áreas onde é mais importante o incremento de matéria orgânica, buscando aumentar a capacidade de troca de cátions efetiva nestas áreas. Planejamento que deve ser auxiliado também pelo mapa do padrão de distribuição espacial da M.O. na área (Figura 3), que identifica os pontos com

Cultura Agronômica, Ilha Solteira, v.26, n.4, p.625-639, 2017 
maior menor quantidade de matéria orgânica. Trabalho conduzidos por SANTOS et al. (2001), com gramíneas e leguminosas mostrou o acúmulo de M.O. e a melhora da capacidade de troca de cátions do solo. Resultados semelhantes foram encontrados por Araújo Júnior (2011), avaliando o incremento relação da capacidade de troca de cátions do solo e o incremento de matéria orgânica em uma lavoura cafeeira região Sul de Minas.
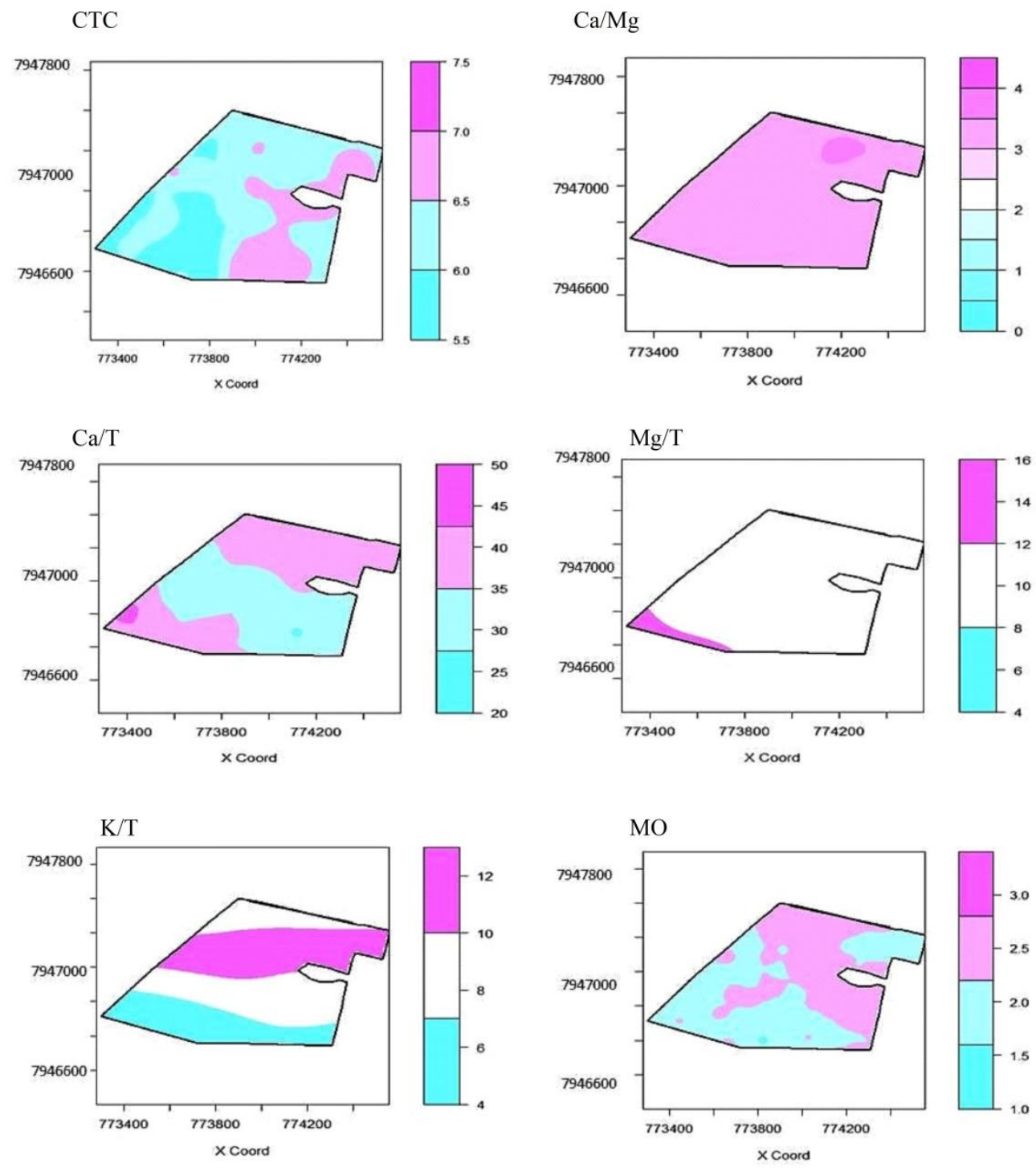

Figura 3. Mapas de padrões espaciais ajustados para a CTC do solo a pH 7,0 (CTC), da relação entre os macronutrientes $(\mathrm{Ca} / \mathrm{Mg} ; \mathrm{Ca} / \mathrm{T} ; \mathrm{Mg} / \mathrm{T}$ e $\mathrm{K} / \mathrm{T})$ e da matéria orgânica do solo (M.O.)

Nos intemperados de maneira geral como é o caso do Latossolos Vermelho-amarelo em estudo, a capacidade de troca de cátions oriunda da matéria orgânica pode representar quase a totalidade destas cargas no solo (NOVAIS et al., 2007); além disso, Bayer e Mielniczuk (2008) relatam que a manutenção ou o aumento do teor de matéria orgânica no solo, é muito importante na diminuição de problemas ambientais como a lixiviação. 
O mapa de padrão espacial da relação $\mathrm{Ca} / \mathrm{Mg}$ (Figura 3), mostra que praticamente não há diferença desta relação na área, ficando próximo a 3:1 que é a relação ótima para o cafeeiro segundo (MATIELLO et al., 2006).

Os mapas da relação do $\mathrm{Ca} / \mathrm{T}, \mathrm{Mg} / \mathrm{T}$ e $\mathrm{K} / \mathrm{T}$ (Figuras 3) caracterizam na área locais onde a relação não está favorável ou favorável, isto possibilita planejar as adubações conforme a necessidade e também a relação deste nutriente na área, visando restabelecer o equilíbrio nestas áreas, podendo assim alcançar o máximo produtivo.

As metodologias de avaliação dos atributos químicos do solo precisam ser aprimoradas, levando em consideração a dependência espacial das variáveis, como o uso de técnicas como a geoestatística. Na atividade cafeeira vem sendo discutida a necessidade em vários estudos como os de Almeida e Guimarães (2016); Lima et al. (2013); Silva et al. (2007); Silva et al. (2010) e Silva e Lima (2012), estudos nos quais observaram a dependência espacial dos atributos do solo.

O estudo do comportamento dos atributos do solo pode permitir uma melhor delimitação das zonas de manejo da propriedade, podendo assim obter de forma rápida e eficaz repostas sobre o próprio manejo no desenvolvimento do cafeeiro. Devendo, portanto, propor recomendações de tratos culturais, buscando otimizar a produção cafeeira.

\section{CONCLUSÃO}

A CTC e a $\mathrm{Ca} / \mathrm{T}$ do solo apresentaram forte dependência espacial, já as relações $\mathrm{Ca} / \mathrm{Mg}, \mathrm{Mg} / \mathrm{T}$ e $\mathrm{K} / \mathrm{T}$, apresentaram moderada dependência espacial.

O maior alcance prático foi da CTC do solo $600 \mathrm{~m}$, o menor da relação $\mathrm{Ca} / \mathrm{Mg}$ de 287,80m, já as relações $\mathrm{Ca} / \mathrm{T}, \mathrm{Mg} / \mathrm{T}$ e $\mathrm{K} / \mathrm{T}$ apresentaram alcances de 581,86, 575,60 e $467,70 \mathrm{~m}$ respectivamente.

\section{REFERÊNCIAS BIBLIOGRÁFICAS}

AGBENIN, J. O.; RAIJ, B. Rate process of calcium, magnesium and potassium desorption from variable-charge soils by mixed ion exchange resins. Geoderma, New York, v. 93, n. 1-2, p.141-157, 1999.

ALMEIDA, L. S., GUIMARÃES, E. C. Geoestatística e análise fatorial exploratória para representação espacial de atributos químicos do solo, na cafeicultura. Coffee Science, Lavras, v. 11, n. 2, p.195 - 203, 2016.

ALVAREZ V., V. H.; NOVAIS, R. F.; BARROS, N. F.; CANTARUTTI, R. B.; LOPES, A. F. Interpretação dos resultados das análises dos solos. In: RIBEIRO, A. C., GUIMARÃES, P. T. G., AlVAREZ V., V. H. (Ed.). Recomendações para o uso de corretivos e fertilizantes em Minas Gerais. $5^{a}$ Aproximação. Viçosa: Comissão de Fertilidade do Solo do Estado de Minas Gerais, 1999. cap. 5, p. 25-32.

Cultura Agronômica, Ilha Solteira, v.26, n.4, p.625-639, 2017 
ARAÚJO JÚNIOR, C. F.; GUIMARÃES, P. T. G.; DIAS JUNIOR, M. S.; ALCANTARA, E. N.; MESNDES, A. D. R. Alterações nos atributos químicos de um latossolo pelo manejo de plantas invasoras em cafeeiros. Revista Brasileira de Ciência Solo, Viçosa, v. 35, n. 6, p.2207-2217, 2011.

BAYER, C.; MIELNICZUK, J. Dinâmica e função da matéria orgânica. In: SANTOS, G.A. SILVA, L. S.; CANELlAS, L. P.; CAMARGO, F. A. O. eds. Fundamentos da matéria orgânica do solo: Eecossistemas tropicais e subbtropicais. 2. ed. Porto Alegre: Metrópole, 2008. cap. 1, p. 7-18.

CAMBARDELLA, C. A.; MOORMAN, T. B.; NOVAK, J. M.; PARKIN, T. B.; KARLEN, D. L.; TURCO, R. F.; KONOPKA, A. E. Field-scale variability of soil proprieties in central Iowa soils. Soil Science Society America Journal, Medison, v. 58, n. 5, p.1240-1248, 1994.

COMPANHIA NACIONAL DE ABASTECIMENTO - CONAB. Estimativas de Safras Agrícolas. Primeiro Levantamento Café 2017. Disponível em: http://www.conab.gov.br/. Acesso em: 24 mar. 2017.

CONSELHO DOS EXPORTADORES DE CAFÉ - CECAFE - Exportações Brasileiras de café. Exportações entre 01/01/2016 a 31/12/2016. Disponível em: http://www.cecafe.com.br/dados-estatisticos/exportacoes-brasileiras/. Acesso em: 24 mar. 2017.

CARVAlHO, D. O.; POZZA, E. A.; CASElA, C. R.; COSTA, R. V.; POZZA, A. A. A.; CARVALHO, C. O. Adubação nitrogenada e potássica na severidade da antracnose em dois cultivares de milho. Revista Ceres, Viçosa, v. 60, n. 3, p.380-387, 2013.

CARVALHO, C. G. P.; FARIAS, C. A. A.; TOLEDO, J. F. F.; ALMEIDA, L. A.; KIIHL, R. A. S.; OLIVEIRA, M. F.; HIROMOTO, D. M.; TAKEDA, C. Proposta de classificação dos coeficientes de variação em relação à produtividade e altura da planta de soja. Pesquisa Agropecuária Brasileira, Brasília, v. 38, n. 2, p.187-193, 2003.

CAVAlCANTE, E. G. S. ALVES, M. C.; PEREIRA, G. T. S. ZIGOMAR M. Variabilidade espacial de MO, P, K e CTC do solo sob diferentes usos e manejos. Ciência Rural, Santa Maria, v. 37, n. 2, p.394-400, 2007.

EMBRAPA. Manual de métodos de análises de solo. 2. ed. Rio de Janeiro: Centro Nacional de Pesquisa de Solos, 2011. 230 p.

FONTES, P. C. R.; PEREIRA, P. R. G. Nutrição mineral do tomate para mesa. Informe Agropecuário, Belo Horizonte, v. 24, n. 219, p.27-34, 2003.

GOMES, F. P. A estatística moderna na pesquisa agropecuária. Piracicaba: Potafós, 1984. $160 \mathrm{p}$.

Cultura Agronômica, Ilha Solteira, v.26, n.4, p.625-639, 2017 
KRAJEWSKI, S. A.; GIBBS, B. L. Uderstanding contouring a pratical guide to spatial estimation using computer and variogram interpretation. Gibbs Associations: Boulder. CO, 2001. $100 \mathrm{p}$.

KÖPPEN, W. Climatologia: con un estudio de los climas de la tierra. México: Fondo de Cultura Económica, 1948. 479 p.

LIMA, J. S. S.; SOUZA, G. S.; SILVA, S. A. Amostragem e variabilidade espacial de atributos químicos do solo em área de vegetação natural em regeneração. Revista Árvore, Viçosa, v. 34, n. 1, p.127-136, 2010.

LIMA, J. S. S.; SILVA, S. A.; SILVA, J. M. Variabilidade espacial de atributos químicos de um Latossolo Vermelho-Amarelo cultivado em plantio direto. Revista Ciência Agronômica, Fortaleza, v. 44, n. 1, p.16-23, 2013.

LOPES, A. S.; GUILHERME, L. R. G., Fertilidade do Solo e Produtividade Agrícola. In: NOVAIS, R. F.; ALVAREZ V., V. H.; BARROS, N. F.; FONTES, R. L. F.; CANTARUTTI, R. B.; NEVES, J. C. L.(Eds.). Fertilidade do solo. Viçosa. Sociedade Brasileira de Ciências do Solo, 2007. cap. 1, p. 1-64.

MATHERON, G. Principles of geostatictics. Economic Geology, Littleton, v. 58, n. 8, p.1246-1266, 1963.

MATIELLO, J. B; GARCIA, A. W. R; ALMEIDA, S. R. Teores de Ca, Mg e K no solo e sua influência no desequilíbrio nutricional da lavoura cafeeira. In: CONGRESSO BRASILEIRO DE PESQUISAS CAFEEIRAS, 32., 2006, Poços de Caldas. Resumos... Varginha: Fundação Pró Café, 2006. p. 27.

MANZIONE, R. L.; ZIMBACK, C. R. L. Análise Espacial Multivariada Aplicada na Avaliação da Fertilidade do Solo. Engenharia na Agricultura, Viçosa, v. 19, n. 3, p.227235, 2011.

MEDEIROS, J. C.; AlBUlQUERQUE, J. A.; MAFRA, A. L.; ROSA, J. D.; GATIBONI, L. C. Relação cálcio:magnésio do corretivo da acidez do solo na nutrição e no desenvolvimento inicial de plantas de milho em um Cambissolo Húmico Álico. Semina, Londrina, v. 29, n. 4, p.799-806, 2008.

MEURER, E. J.; RHEINHEIMER, D.; BISSANI, C. A. Fenômenos de sorção em solos. In: MEURER, E. J. Fundamentos de Química do Solo. 3.ed. Porto Alegre: EVANGRAF, 2006. cap. 5, p. 117-162.

MOLIN, J. P.; CASTRO, C. N. Establishing management zones using soil electrical conductivity and other soil properties by the fuzzy clustering technique. Scientia Agricola, Piracicaba, v. 65, n. 6, p.567-573, 2008.

NANNI, M. R.; POVH, F. P.; DEMATTÊ, J. A. M.; OLIVEIRA, R. B.; CHICATI, M. L.; CEZAR, E. Optimum size in grid soil sampling for variable rate application in site-specific management. Scientia Agricola, Piracicaba, v. 68, n. 3, p.386-392, 2011. 
NOVAIS, R. F.; ALVAREZ V., V. H.; BARROS, N. F.; FONTES, R. L. F.; CANTARUTTI, R. B.; NEVES, J. C. L. (Eds.). Fertilidade do solo. Viçosa: Sociedade Brasileira de Ciências do Solo, 2007. 432 p.

OLIVEIRA, E. F.; PAVAN, M. A.; CHAVES, J. C. D. Respostas das mudas de cafeeiro ao equilíbrio entre cátions trocáveis em solos com cargas variáveis. Arquivo de Biologia e Tecnologia, v. 37, n. 4, p.973-979, 1994.

OLIVEIRA, L. B. T.; SANTOS, A. C.; LIMA, J. S.; NEVES NETO, D. N. Variabilidade espacial das respostas produtivas e morfológicas do capim-Marandu em função dos atributos químicos e topográficos. Revista Brasileira de Saúde e Produção Animal, Salvador, v. 16, n. 4, p.772-783, 2015.

R DEVELOPMENT CORE TEAM. R: A language and environment for statistical computing. Vienna, Austria: R Foundation for Statistical Computing. Disponível em: http://www.r-project.org. Acesso em: 4 nov. 2015

RIBEIRO JÚNIOR, P. J.; DIGGLE, P. J. GeoR: a package for geostatistical analysis. RNEWS, Pelotas, v. 1, n. 2, p.15-18, 2001.

ROSOLEN, V.; RESENDE, T. M.; BORGES, E. N.; FRAGE, C. T.; MACHADO, H. A. Variações nos teores do $\mathrm{C}$ total e isotópico do solo após substituição do cerrado em sistemas agrícolas no Triângulo Mineiro. Sociedade \& Natureza, Uberlândia, v. 24, n. 1, p.157-168, 2012

SANTOS, A. C.; SILVA, L. F.; LIMA, J. R. S.; ANDRADE, A. P.; CALVACANTE, V. R. Gramíneas e leguminosas na recuperação de áreas degradadas: efeito nas características químicas de solo. Revista Brasileira de Ciência Solo, Viçosa, v. 25, n. 4, p.1063-1071, 2001 .

SILVA, F. M.; SOUZA, Z. M.; FIGUEIREDO, C. A. P.; MARQUES JÚNIOR, J.; MACHADO, R. V. Variabilidade espacial de atributos químicos e de produtividade na cultura do café. Ciência Rural, Santa Maria, v. 37, n. 2, p.401-407, 2007.

SILVA, S. A.; LIMA, J. S. S.; XAVIER, A. C.; TEIXEIRA, M. M. Variabilidade espacial de atributos químicos de um Latossolo Vermelho-Amarelo húmico cultivado com café. Revista Brasileira de Ciência do Solo, Viçosa, v. 34, n. 1, p.15-22, 2010.

SILVA, S. A.; LIMA, J. S. S. Multivariate Analysis and Geostatistics of the Fertility of a Humic Rhodic Hapludox under Coffee Cultivation. Revista Brasileira de Ciência do Solo, Viçosa, v. 36, n. 2, p.467-474, 2012.

SILVA, J. E. Balanço de cálcio e magnésio e desenvolvimento de milho em solos de cerrado. Pesquisa Agropecuária Brasileira, Brasília, v. 15, n. 3, p.329-333, 1980.

SPARKS, D. L. Inorganic soil components. In: SPARKS, D. L. (ed.). Environmental soil chemistry. San Diego: Academic Press, 1995. cap. 2, p. 23-52.

Cultura Agronômica, Ilha Solteira, v.26, n.4, p.625-639, 2017 
SOARES, A. Geoestatística para ciências da terra e do ambiente. 2. ed. Lisboa: IST Press, 2006. 2014 p.

SOUZA, G. S.; LIMA, J. S. S.; SILVA, S. A.; OLIVEIRA, R. B. Variabilidade espacial de atributos químicos em um Argissolo sob pastagem. Acta Scientiarum Agronomy, Maringá, v. 30, n. 4, p.589-596, 2008.

SOUZA, P. A.; VENTURIN, N.; MACEDO, R. L. G. Adubação Mineral do Ipê-Roxo (Tabebuia impetiginosa). Ciência Florestal, Santa Maria, v. 16, n. 3, p.261-270, 2006.

VIEIRA, S. R.; HATFIELD, J. L.; NIELSEN, D. R.; BIGGAR, J. W. Geostatistical theory and application to variability of some agronomical properties. Hilgardia, Berkeley, v. 31, n. 3, p.01-75, 1983.

YAMAMOTO, J. K.; LANDIM, P. M. B. Geoestatística: conceitos e aplicações. 1. ed. São Paulo: Ed. Oficina de Textos, 2013. 215p. 